\title{
INFINITE DIMENSIONAL REPRESENTATIONS OF $\tilde{D}_{4}$
}

\author{
by A. DEAN and F. ZORZITTO
}

(Received 27 August, 1988)

Introduction. By a representation of the extended Dynkin diagram $\tilde{D}_{4}$, we shall mean a list of 5 vector spaces $P, E_{1}, E_{2}, E_{3}, E_{4}$ over an algebraically closed field $K$, and 4 linear maps $a_{1}, a_{2}, a_{3}, a_{4}$ as shown.

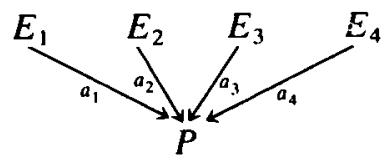

The spaces need not be of finite dimension.

In their solution of the 4-subspace problem [6], Gelfand and Ponomarev have classified such representations when the spaces are finite dimensional. A representation like (1) can also be viewed as a module over the $K$-algebra $R_{4}$ consisting of all $5 \times 5$ matrices having zeros off the first row and off the main diagonal.

The algebra $R_{4}$ is an interesting example of a tame, hereditary, finite dimensional algebra. A general theory of infinite dimensional representations of tame, hereditary algebras $\Lambda$ was developed by $C$. M. Ringel in [11]. Most of the terminology that we use may be found in Ringel. In particular, this includes notions of purity, torsion freeness, rank and regularity. In [11, §6], we find a classification of all torsion free $\Lambda$-modules of rank 1 , including those of infinite dimension over the field $K$. However the infinite dimensional, torsion free, indecomposable modules of rank 2 or more require further investigation. Especially, how are they to be constructed? What interesting isomorphism invariants do they possess? It seems that examples of such $\Lambda$-modules are needed.

In this note we shall construct a family of infinite dimensional, torsion free, indecomposable $R_{4}$-modules of rank 2 , each having no proper pure submodule. Such purely simple modules have been constructed in total for the Kronecker algebra $A=\left[\begin{array}{cc}K & K^{2} \\ 0 & K\end{array}\right]$ by use of some exotic $K$-linear functionals on the space $K(x)$ of rational functions in the indeterminate $x$, see e.g. [3], [5], [8]. Our approach for $R_{4}$ vaguely resembles the ones for the algebra $A$. We expect that a general method for constructing all purely simple $\Lambda$-modules of finite rank may evolve from these methods, at least when the ground field $K$ is algebraically closed.

Our paper is in two parts. The first part deals with a general criterion (Theorem 2.6) for pure simplicity of an infinite dimensional, torsion free, finite rank $\Lambda$-module, where $\Lambda$ is any tame, hereditary, finite dimensional algebra. In passing we obtain a structural result for purely simple modules of rank 2 (Theorem 2.5). This theorem extends Okoh's result [10, Proposition B] for the rank 2 case to all tame, hereditary algebras. Theorem 2.5 also suggests that the purely simple modules of rank 2 form a tractable part of the class of indecomposables.

In the second part, we use the general criterion in the construction of the infinite dimensional, purely simple $R_{4}$-modules of rank 2 . When dealing with $R_{4}$-modules, we

Glasgow Math. J. 32 (1990) 25-33. 
shall take the approach of (1). In particular a homomorphism, as shown

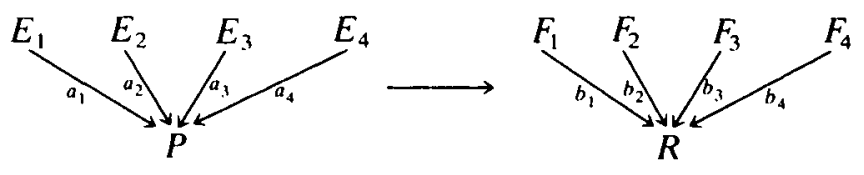

between two $R_{4}$-modules, is a list of 5 linear maps

$$
P \stackrel{\varphi}{\longrightarrow} R, \quad E_{1} \stackrel{\varphi_{1}}{\longrightarrow} F_{1}, \quad E_{2} \stackrel{\varphi_{2}}{\longrightarrow} F_{2}, \quad E_{3} \stackrel{\varphi_{3}}{\longrightarrow} F_{3}, \quad E_{4} \stackrel{\varphi_{4}}{\longrightarrow} F_{4}
$$

such that

$$
\varphi \circ a_{i}=b_{i} \circ \varphi_{i}
$$

for $i=1,2,3,4$. All of the homomorphisms between the $R_{4}$-modules in the second part will be displayed as such lists of 5 linear maps.

In [3, Theorem 2.8], it is shown that one can obtain examples of infinite dimensional, purely simple $R_{4}$-modules of arbitrary finite rank by constructing Kronecker modules with the same properties and then applying a certain functor from Kronecker modules to $R_{4}$-modules. The advantages of the approach which is taken in this paper are twofold. First, the construction procedure is self-contained within the category of $R_{4}$-modules. Secondly, it gives a method for obtaining all infinite dimensional purely simple $R_{4}$-modules of rank 2 and not just those which are images of rank 2 Kronecker modules.

A homomorphism test for pure simplicity. The results of this section are valid for modules over any tame, hereditary, finite dimensional algebra $\Lambda$. Ringel's paper [11] provides a good background to the definitions and concepts which we will use. However, for the sake of completeness we include some of these definitions.

Let $M$ be a finite dimensional right $N$-module, and let $0 \rightarrow P_{1} \stackrel{f}{\rightarrow} P_{2} \rightarrow M \rightarrow 0$ be the complete minimal projective resolution of $M$ (the map $f$ is a monomorphism because $\Lambda$ is hereditary). Applying the functor $*=\operatorname{Hom}_{\Lambda}\left(, \Lambda_{\Lambda}\right)$, we obtain a map $f^{*}: P_{2}^{*} \rightarrow P_{1}^{*}$ of left $\Lambda$-modules. The cokernel of this map is denoted by $\operatorname{Tr} M$. Similarly, starting with a finite dimensional left $\Lambda$-module $N$, and its complete minimal projective resolution, we apply $*=\operatorname{Hom}_{\Lambda}\left(,{ }_{\Lambda} \Lambda\right)$, and obtain as cokernel a right $\Lambda$-module denoted by $\operatorname{Tr} N$. Now, using the duality functor $D=\operatorname{Hom}_{K}(, K)$, we obtain, from the left $\Lambda$-module $\operatorname{Tr} M$, a right $\Lambda$-module $A M=D \operatorname{Tr} M$. If we first apply $D$ to the right module $M$ and then apply $\operatorname{Tr}$ to the left module $D M$, we obtain the right module $A^{-1} M=\operatorname{Tr} D M$. Let $X$ be a finite dimensional right $\Lambda$-module. We say that $X$ is preprojective if $X$ is isomorphic to $A^{-i} P$ for some non-negative integer $i$ and some indecomposable projective module $P$. We say that $X$ is preinjective if $X$ is isomorphic to $A^{i} I$ for some non-negative integer $i$ and some indecomposable injective module $I$. A module is called regular provided it has no indecomposable preprojective or preinjective direct summands.

Given a $\Lambda$-module $X$, let $\xi X$ be the sum of all finite dimensional submodules $U$ of $X$ such that $U$ has no indecomposable preprojective direct summand. Call $X$ torsion provided $\zeta X=X$, and torsion free if $\zeta X=0$.

A module $Y$ is called divisible provided $\operatorname{Ext}_{\Lambda}(X, Y)=0$ for every simple regular 
module $X$. By simple regular, we mean a regular module which does not have any proper, non-zero regular submodules.

There exists a unique indecomposable, torsion free, divisible $\Lambda$-module which we denote by $Q$, see [11, 5.3]. This module is important for investigating infinite dimensional, torsion free $\Lambda$-modules. The module $Q$ can be characterized in a different way; it is the only infinite dimensional $\Lambda$-module whose endomorphism ring is a division ring and which is finite dimensional as a vector space over its endomorphism ring, [11, $5.3,5.7]$. This characterization of $Q$ is used by Ringel in [12].

Any torsion free $\Lambda$-module $X$ can be embedded into a direct sum $Y$ of copies of $Q$ such that $Y / X$ is torsion regular. The number of copies of $Q$ in the direct sum $Y$ is an invariant of $X$ and is called the rank of $X$ (see [11, 5.5]). Using [11, 5.1], we can easily show that any two embeddings of $X$ into $Y$ with torsion regular quotient are equivalent up to an automorphism of $Y$. As a result, we will speak about the embedding of $X$ into $Y$ with torsion regular quotient. Our first observation is that the embedding of $X$ into $Y$ with torsion regular quotient determines all homomorphisms from $X$ to $Q$. The proof of this proposition follows immediately from [11, 4.7 Corollary].

Proposition 2.1. Let $X$ and $Y$ be as above and let $\alpha: X \rightarrow Y$ be the embedding with torsion regular quotient. Then any homomorphism $\omega: X \rightarrow Q$ factors through $\alpha$.

We shall need to know that the notion of rank is additive in short exact sequences.

Proposition 2.2. Suppose that $0 \rightarrow P \stackrel{\beta}{\rightarrow} X \stackrel{\gamma}{\rightarrow} F \rightarrow 0$ is a short exact sequence of torsion free $\Lambda$-modules. Then $\operatorname{rank} X=\operatorname{rank} P+\operatorname{rank} F$.

Proof. Let $\alpha_{1}: P \rightarrow Y_{1}, \alpha_{2}: F \rightarrow Y_{2}$ be the embeddings of $P$ and $F$ into direct sums $Y_{1}$ and $Y_{2}$ of copies of $Q$, with torsion regular quotients. From the definition of rank it suffices to give an embedding $\alpha: X \rightarrow Y_{1} \oplus Y_{2}$ with torsion regular quotient.

By [11, 4.7 Corollary], there is a map $\delta: X \rightarrow Y_{1}$ such that $\alpha_{1}=\delta \circ \beta$. Define $\alpha: X \rightarrow Y_{1} \oplus Y_{2}$ by $x \rightarrow\left(\delta(x), \alpha_{2} \circ \gamma(x)\right)$. If $\alpha(x)=(0,0)$ for some $x$ in $X$ then $\gamma(x)=0$ since $\alpha_{2}$ is an embedding. Thus $x=\beta(p)$ for some $p$ in $P$. Then $0=\delta(x)=\delta \circ \beta(p)=$ $\alpha_{1}(p)$ forces $p$, and $x$, to be 0 because $\alpha_{1}$ is an embedding. Hence $\alpha$ is an embedding.

We define a map $Y_{1} / \alpha_{1}(P) \rightarrow\left(Y_{1} \oplus Y_{2}\right) / \alpha(x)$ by $y_{1}+\alpha_{1}(P) \rightarrow\left(y_{1}, 0\right)+\alpha(X)$. This is well defined. So is the map $\left(Y_{1} \oplus Y_{2}\right) / \alpha(X) \rightarrow Y_{2} / \alpha_{2}(F)$ given by $\left(y_{1}, y_{2}\right)+\alpha(X) \rightarrow$ $y_{2}+\alpha_{2}(F)$. The diagram below commutes.

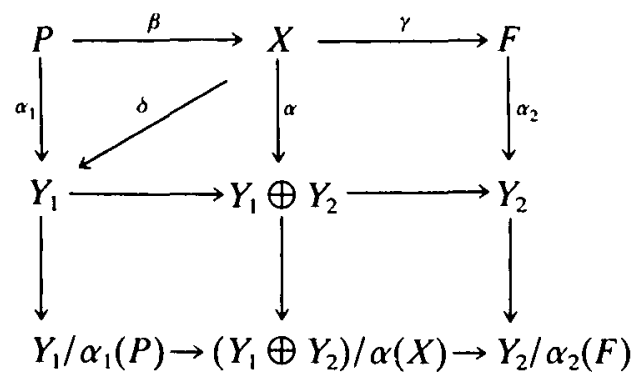

Since all three columns of the diagram and the top two rows are short exact, the bottom row is short exact. But $Y_{1} / \alpha_{1}(P)$ and $Y_{2} / \alpha_{2}(F)$ are torsion regular. By $[11,4.1,4.2]$, the torsion regular modules are closed under extensions. Thus $\left(Y_{1} \oplus Y_{2}\right) / \alpha(X)$ is torsion regular, and $\alpha$ fulfills the requirements. 
We are interested in the class of infinite dimensional $\Lambda$-modules which are purely simple of finite rank. A module is purely simple if it does not have any proper, non-zero pure submodules. Here pure is used in the sense of $P$. M. Cohn [2]; also see [11, $\S \mathrm{F}]$. Unlike the more restrictive notion of direct summand, that of a pure submodule has led to a fruitful analysis of infinite dimensional modules. In the case of finite dimensions, pure simplicity coincides with indecomposability.

We shall say that a submodule $M$ of a module $X$ is torsion closed in $X$ if $X / M$ is torsion free.

Proposition 2.3. Let $M$ be a pure submodule of a torsion free module $X$. Then $M$ is torsion closed in $X$.

Proof. According to [11, 4.1], a module is torsion free provided every finite dimensional, indecomposable submodule is preprojective. Let $U / M$ be a finite dimensional, indecomposable submodule of $X / M$. Since $M$ is pure in $X, M$ is a direct summand in $U$. Let $N$ be a direct complement of $M$ in $U$. Then $N$, being isomorphic to $U / M$, is finite dimensional and indecomposable. Since $X$ is torsion free, $N$ is preprojective and thus $U / M$ is preprojective.

Proposition 2.3 confirms that torsion free modules of rank 1 are purely simple. Indeed if $X$ is a torsion free module with a proper, non-zero, pure submodule $P$ then Proposition 2.2 applied to the short exact sequence $0 \rightarrow P \rightarrow X \rightarrow X / P \rightarrow 0$ forces rank $X \geq 2$.

The next theorem has been proved by Okoh for modules over the Kronecker algebra $A$ (see [8, Lemma 1.12]). Using [11, 2.2 Corollary 3, 6.1 Proposition] it is possible for us to imitate Okoh's proof to cover the general setting.

TheOREM 2.4. Let $X$ be a purely simple $\Lambda$-module. Then any proper, torsion closed submodule which is of finite rank must be finite dimensional.

The above results yield an interesting structural property of purely simple modules of rank 2 .

THEOREM 2.5. If $X$ is a purely simple module of rank 2, then either

(i) every non-zero homomorphism $X \rightarrow Q$ is an embedding or

(ii) $X$ sits in an extension $0 \rightarrow P \rightarrow X \rightarrow F \rightarrow 0$, where $P$ and $F$ are torsion free of rank 1 and $P$ is finite dimensional.

Proof. We note that situations (i) and (ii) are mutually exclusive because $F$ embeds in $Q$ and the map $X \rightarrow F$ in the extension is a non-zero, non-monic homomorphism.

Now there always exists a non-zero map $X \rightarrow Q$. For instance, take the embedding $X \rightarrow Q \oplus Q$ with torsion regular quotient and follow it by a projection onto one of the components $Q$ in the direct sum. If (i) fails then there is a non-zero homomorphism $\varphi: X \rightarrow Q$ with a non-zero kernel. Let $P=\operatorname{ker} \varphi, F=$ image $\varphi$. We have the exact sequence $0 \rightarrow P \rightarrow X \rightarrow F \rightarrow 0$. By Theorem 2.2, $P$ and $F$ have rank 1 and, by Theorem 2.4, $P$ is finite dimensional.

In the case of the Kronecker algebra $A$, condition (i) of Theorem 2.5 does not occur (see [10, Proposition $\mathrm{B}]$ ). However, for the algebra $R_{4}$, (i) does occur. Indeed, there exist 
simple projective modules $X$ of rank 2, and these cannot satisfy (ii). In Section 3 we construct examples of purely simple, infinite dimensional $R_{\mathbf{4}}$-modules that fall into the situation of (ii). However, it would be interesting to know if there exist purely simple $R_{\mathbf{4}}$-modules of rank 2 that are infinite dimensional and satisfy (i).

We are now ready to present our main result.

THEOREM 2.6. A torsion free module $X$ of finite rank and infinite dimension is purely simple if and only if every non-zero homomorphism $X \rightarrow Q$ has a finite dimensional kernel.

Proof. Suppose there exists a non-zero homomorphism $X \rightarrow Q$ with infinite dimensional kernel. Since $Q$ is torsion free, the kernel is a proper torsion closed submodule. By Proposition 2.2 and Theorem $2.4, X$ is not purely simple.

Conversely, suppose that $X$ is not purely simple. Let $M$ be a proper pure submodule of $X$. If $M$ is finite dimensional then it is a direct summand in $X$ by [11, $\S \mathrm{F}]$, and its complement is a proper, infinite dimensional, pure submodule of $X$. As a result, we see that $X$ has a proper, infinite dimensional, pure submodule. Let $L$ be such a submodule. By Proposition 2.3, $L$ is torsion closed in $X$. Since $L$ is proper, $\operatorname{rank} L<\operatorname{rank} X$. Let $m=\operatorname{rank} X-\operatorname{rank} L$. The quotient module $X / L$ is torsion free of rank $m$. Let $\alpha$ be the embedding of $X / L$ into $m$ copies of $Q$ such that the quotient is torsion regular. If we follow $\alpha$ by any one of the canonical projections onto $Q$, we have a non-zero homomorphism $\beta: X / L \rightarrow Q$. The canonical projection of $X$ onto $X / L$ followed by $\beta$ yields the required homomorphism from $X$ to $Q$.

Purely simple representations of $\tilde{D}_{4}$. For the representations of $\tilde{D}_{4}$, i.e. $R_{4^{-}}$ modules, we take the point of view given by (1). The ground field in this section needs to be algebraically closed.

We will construct explicitly a family of purely simple $R_{4}$-modules that are infinite dimensional and of rank 2 . Each will be an extensions of a torsion free, finite dimensional module $P$ of rank one by an infinite dimensional module $F$ of rank one, as in Theorem 2.5(ii).

The module $F$ that we shall use is the unique indecomposable, torsion free, divisible module $Q$. For $\tilde{D}_{4}$, the module $Q$ is

with action given by

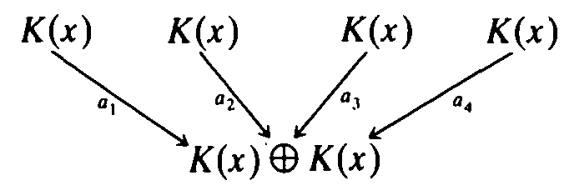

$$
a_{1}(r)=(r, r), \quad a_{2}(r)=(r, x r), \quad a_{3}(r)=(r, 0), \quad a_{4}(r)=(0, r) .
$$

Here $K(x)$ is the space of rational functions in one variable $x$.

To show that (3) gives the unique torsion free, divisible, indecomposable module, one has two approaches. The first is to calculate the endomorphism ring of $Q$. Such a computation yields that End $Q=K(x)$. A rational function $t$ furnishes an endomorphism of (3) by multiplying each of the 5 component spaces of (3) by $t$. Since $\operatorname{dim}_{\text {End } Q} Q=6$, we are done by the comments about $Q$ at the beginning of the previous section. 
The second approach is more circuitous. There exists a functor $T$ from the category of Kronecker modules to the category of $R_{4}$-modules (see [4]). One of the properties of this functor is that it maps the unique torsion free, indecomposable divisible Kroncker module onto the unique torsion free, indecomposable divisible $R_{4}$-module by [3, Theorem 2.8].

The torsion free, indecomposable, divisible Kronecker module is described in Definition 9.6 of [1]. Applying $T$ to that module gives us (3).

For the module $P$, take

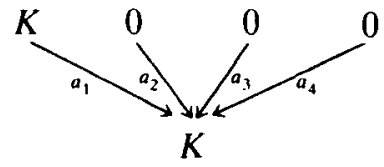

with $a_{1}$ the identity and the other maps 0 . This $P$ given by (4) is clearly indecomposable and torsion free, in fact projective. The defect of $P$ is -1 , and hence, by $[11,5.6]$, the rank of $P$ is 1 . See [4] for a definition of defect. the form

We will be looking at modules $X$ which lie in the middle of short exact sequences of

$$
0 \rightarrow P \stackrel{a}{\rightarrow} X \stackrel{\tau}{\rightarrow} Q \rightarrow 0 .
$$

In order to construct the modules, we need $K$-linear functionals $f: K(x) \rightarrow K$. For each functional $f$, let $X$ be the module

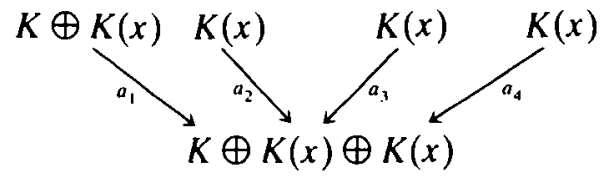

with action given by

$$
\begin{array}{ll}
a_{1}(\lambda, r)=(\lambda, r, r), & a_{2}(r)=(f(r), r, x r) \\
a_{3}(r)=(0, r, 0), & a_{4}(r)=(0,0, r)
\end{array}
$$

for all $\lambda$ in $K, r$ in $K(x)$. This module $X$ is the middle term of a short exact sequence (5) with obvious maps $\sigma$ and $\tau$.

Using techniques similar to those in [10], one can show that every extension $X$ of $P$ by $Q$, as in (5), is equivalent to (6) for some functional $f$. However we will leave this aside.

In order to determine necessary and sufficient conditions on the functional $f$ for the module $X$ to be purely simple, we need the derived function that was introduced in [7, $\S 3]$. For each functional $f$, the derived function is a $K$-linear map $\partial_{f}: K(x) \rightarrow K(x)$ with the properties that

$$
\partial_{f}(1)=0 \quad \text { and } \quad \partial_{f}(x r)=x \partial_{f}(r)+f(r)
$$

for all $r$ in $K(x)$.

The rank of $P$ is 1 , and the embedding $\rho: P \rightarrow Q$ with torsion regular quotient is 
given by the 5 linear maps:

$$
\begin{aligned}
K \rightarrow K(x)+K(x), & \text { where } \lambda \rightarrow(\lambda, \lambda), \quad K \rightarrow K(x), \quad \text { where } \lambda \rightarrow \lambda, \\
0 \rightarrow K(x), \quad 0 \rightarrow K(x), \quad 0 \rightarrow K(x) . &
\end{aligned}
$$

According to [11, 4.7 Corollary], there is a module map $\epsilon: X \rightarrow Q$ such that $\rho=\epsilon \sigma$. By using (7), we can check that $\epsilon$ is given by the following list of 5 linear maps:

where

$$
K \oplus K(x) \oplus K(x) \rightarrow K(x) \oplus K(x),
$$

$$
\begin{gathered}
\left(\lambda, r_{1}, r_{2}\right) \rightarrow\left(\lambda+(x-1) \partial_{f}\left(r_{1}\right), \lambda+(x-1) \partial_{f}\left(r_{2}\right)\right), \\
K \oplus K(x) \rightarrow K(x), \quad \text { where }(\lambda, r) \rightarrow \lambda+(x-1) \partial_{f}(r), \\
K(x) \rightarrow K(x), \quad \text { where } r \rightarrow f(r)+(x-1) \partial_{f}(r), \\
K(x) \rightarrow K(x), \quad \text { where } r \rightarrow(x-1) \partial_{f}(r), \\
K(x) \rightarrow K(x), \quad \text { where } r \rightarrow(x-1) \partial_{f}(r) .
\end{gathered}
$$

As in the proof of Proposition 2.2, there results from this $\epsilon$ a module map $\alpha: X \rightarrow Q \oplus Q$ with torsion regular quotient. The following list of 5 maps gives $\alpha$ :

where

$$
K \oplus K(x) \oplus K(x) \rightarrow(K(x) \oplus K(x)) \oplus(K(x) \oplus K(x)),
$$

$$
\begin{array}{rll}
\left(\lambda, r_{1}, r_{2}\right) \rightarrow\left(\lambda+(x-1) \partial_{f}\left(r_{1}\right), \lambda+(x-1) \partial_{f}\left(r_{2}\right), r_{1}, r_{2}\right), & \\
K \oplus K(x) \rightarrow K(x) \oplus K(x), & \text { where } & (\lambda, r) \rightarrow\left(\lambda+(x-1) \partial_{f}(r), r\right), \\
K(x) & \rightarrow K(x) \oplus K(x), \quad \text { where } & r \rightarrow\left(f(r)+(x-1) \partial_{f}(r), r\right), \\
K(x) & \rightarrow K(x) \oplus K(x), \quad \text { where } & r \rightarrow\left((x-1) \partial_{f}(r), r\right), \\
K(x) & \rightarrow K(x) \oplus K(x), \quad \text { where } & \left.r \rightarrow\left((x-1) \partial_{f}(r), r\right)\right) .
\end{array}
$$

According to Theorem 2.6, we are interested in what conditions $f$ must satisfy so that every non-zero homomorphism $v: X \rightarrow Q$ has a finite dimensional kernel. Using Proposition 2.1, any homomorphism $v: X \rightarrow Q$ factors through the embedding (8). Since End $Q=K(x)$, it follows that every map $Q \oplus Q \rightarrow Q$, and hence $v$, arises from two rational functions $s$ and $t$. The map $v$ will be zero if and only if both $s=0$ and $t=0$. Given the nature of the embedding $\alpha$ in (8), a homomorphism $v: X \rightarrow Q$ is thus defined by the following list of 5 linear maps:

where

$$
K \oplus K(x) \oplus K(x) \rightarrow K(x) \oplus K(x),
$$

$$
\begin{aligned}
\left(\lambda, r_{1}, r_{2}\right) & \rightarrow\left(s\left(\lambda+(x-1) \partial_{f}\left(r_{1}\right)+t r_{1}, s\left(\lambda+(x-1) \partial_{f}\left(r_{2}\right)\right)+t r_{2}\right),\right. \\
K \oplus K(x) & \rightarrow K(x), \quad \text { where } \quad(\lambda, r) \rightarrow s\left(\lambda+(x-1) \partial_{f}(r)\right)+t r, \\
K(x) & \rightarrow K(x), \quad \text { where } r \rightarrow s\left(f(r)+(x-1) \partial_{f}(r)\right)+t r, \\
K(x) & \rightarrow K(x), \quad \text { where } r \rightarrow s(x-1) \partial_{f}(r)+t r, \\
K(x) & \rightarrow K(x), \quad \text { where } r \rightarrow s(x-1) \partial_{f}(r)+t r .
\end{aligned}
$$

Now ker $v$ is infinite dimensional if and only if one of the 5 linear maps in (9) has an infinite dimensional kernel. No matter which one it is, this is tantamount to saying that

$$
s(x-1) \partial_{f}(r)+t r=0
$$


for infinitely many linearly independent $r$ in $K(x)$. For instance, if the second map in the list had an infinite dimensional kernel then the restriction of that map to $0 \oplus K(x)$ would still have an infinite dimensional kernei, thereby giving (10) for infinitely many linearly independent $r$.

Hence $X$ is purely simple if and only if for every non-zero choice of rational functions $s, t$, equation (10) is satisfied only on a finite dimensional space of rational functions $r$. According to [3, Theorem 2.5], this is equivalent to having the following conditions hold:

(a) for every $\theta$ in $K$ the power series $\sum_{k=1}^{\infty} f\left((x-\theta)^{-k}\right) x^{k}$ is not the expansion of a rational function, nor is the series $\sum_{k=0}^{\infty} f\left(x^{k}\right) x^{k}$,

(b) for any rational function $r$ the set $\left\{\theta \in K: f\left((x-\theta)^{-1}\right)=r(\theta)\right\}$ is finite.

The algebraic closure of $K$ was needed to have these conditions. In particular it is necessary to know that the functions $(x-\theta)^{-k}, k=1,2, \ldots$, and $x^{k}, k=0,1, \ldots$, form a basis of $K(x)$ over $K$.

For an example of such a functional $f$, let $\left(n_{1}, n_{2}, n_{3}, \ldots\right)$ be the sequence $(1,0,1,0$, $0,1,0,0,0,1,0,0,0,0,1, \ldots)$ and let $\vee \theta$ be a square root of $\theta$ for each $\theta$ in $K$. Then define $f$ on the basis of $K(x)$ by: $f\left(x^{k}\right)=n_{k}, k=0,1,2, \ldots$, and, for $\theta$ in $K$, $f\left((x-\theta)^{-1}\right)=\sqrt{ } \theta, f\left((x-\theta)^{-k}\right)=n_{k}, k=2,3, \ldots$ This $f$, and many other like it, satisfy both (a) and (b) yielding a purely simple representation of $X$ of $D_{4}$.

The above construction of a rank 2 example can also be imitated to create examples of purely simple $R_{4}$-modules of any finite rank.

Finally we observe that rank 2 , purely simple $R_{4}$-modules exist in abundance.

Proposition 3.1. Every infinite dimensional, torsion free, rank $1 R_{4}$-module is a quotient of a purely simple rank 2 module.

Proof. Let $F$ be an infinite dimensional, torsion free, rank $1 R_{4}$-module and let $X$ be the purely simple rank 2 module constructed just above. Let $\sigma: F \rightarrow Q$ be the embedding with torsion regular quotient. We construct the pullback $N$ as in the following diagram.

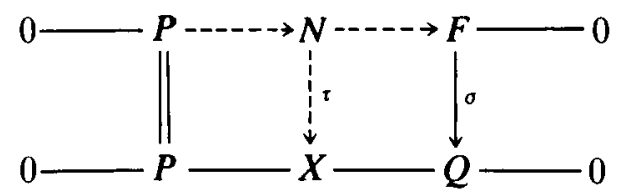

The module $N$ is an infinite dimensional rank 2 module. The cokernel of $\tau$ is isomorphic to the cokernel of $\sigma$ and hence is torsion regular. If $N$ is not purely simple then, by Theorem 2.6, there exists a non-zero homomorphism $N \rightarrow Q$ with infinite dimensional kernel. By [11, 4.7 Corollary], this homomorphhism would extend to $X$. Again by Theorem 2.6,this implies that $X$ is not purely simple. This is a contradiction and so $N$ is purely simple.

\section{REFERENCES} 273-338.

1. N. Aronszajn and U. Fixman, Algebraic spectral problems, Studia Math. 30 (1968),

2. P. M. Cohn, On the free product of associative rings, Math. Z. 71 (1959), 380-398. 


\section{REPRESENTATIONS OF $\tilde{D}_{4}$}

3. A. Dean and F. Zorzitto, A criterion for pure simplicity, to appear in J. Algebra.

4. V. Dlab and C. M. Ringel, Indecomposable representations of graphs and algebras, Mem. Amer. Math. Soc. 173 (1976).

5. U. Fixman, On algebraic equivalence between pairs of linear transformations, Trans. Amer. Math. Soc. 113 (1964), 424-453.

6. I. M. Gel'fand and V. A. Ponomarev, Quadruples of subspaces of a finite dimensional space, Soviet Math. Dokl. 12 (1971), 535-539.

7. J. Lawrence, F. Okoh and F. Zorzitto, Rational functions and Kronecker modules, Comm. Algebra 14 (1986), 1947-1965.

8. F. Okoh, A bound on the rank of purely simple systems, Trans. Amer. Math. Soc. 232 (1977), 169-186.

9. F. Okoh, Some properties of purely simple Kronecker modules I, J. Pure Appl. Algebra 27 (1983), 39-48.

10. F. Okoh, Applications of linear functionals to Kronecker modules I, Linear Algebra Appl. 76 (1986), 165-204.

11. C. M. Ringel, Infinite-dimensional representations of finite-dimensional hereditary algebras, Symp. Math. 23 (1979), 321-412.

12. C. M. Ringel, The spectrum of a finite-dimensional algebra, Ring theory (Proc. Antwerp Conf., 1978), Lecture Notes in Pure and Appl. Math. 51 (Dekker, 1979), 535-597.

Department of Mathematics

BISHOP'S UNIVERSITY

LENNOXVILLE, QUEBEC

CANADA J1M $1 Z 7$
Department of Pure Mathematics UNIVERSTTY OF WATERLOO

WATERLOO, ONTARIO

CANADA N2L 3G1 